\title{
Serum Uric Acid to HDL-Cholesterol Ratio is Associated with New-Onset NAFLD in Lean Chinese People with Normal Blood Lipid Levels: a Longitudinal Cohort Study
}

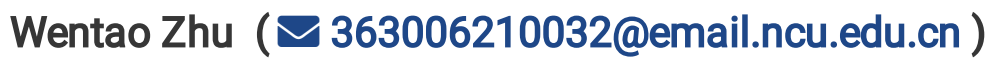
the First Affiliated Hospital of Nanchang University

An Liang the First Affiliated Hospital of Nanchang University

Pei Shi the First Affiliated Hospital of Nanchang University

Song Yuan

the First Affiliated Hospital of Nanchang University

Ying Zhu the First Affiliated Hospital of Nanchang University

Jiwei Fu the First Affiliated Hospital of Nanchang University

Ting Zheng the First Affiliated Hospital of Nanchang University

\section{Zhilong Wen}

the First Affiliated Hospital of Nanchang University

Xiaoping Wu

the First Affiliated Hospital of Nanchang University

\section{Research Article}

Keywords: Lean, Longitudinal study, UHR, UA/HDL-cholesterol ratio, Non-alcoholic fatty liver disease Posted Date: December 2nd, 2021

DOI: https://doi.org/10.21203/rs.3.rs-1117643/v1

License: (1) This work is licensed under a Creative Commons Attribution 4.0 International License. Read Full License 


\section{Abstract}

Background: Nonalcoholic fatty liver disease (NAFLD) is commonly occurred in the non-obese individuals. The serum uric acid (UA) to high-density lipoprotein cholesterol (HDL-c) ratio (UHR) is considered as a predictive factor of NAFLD. However, it is still difficult to confirm the correlation in lean Chinese people with normal blood lipid levels. It is aimed to analyze the correlation of UHR with NAFLD among lean Chinese population without dyslipidemia and compared UHR with other predictors in this study.

Methods: 9838 lean people without NAFLD were included in a retrospective cohort study. NAFLD was diagnosed by liver ultrasound.

Results: A total of 9,838 lean patients with normal blood lipid levels were included in the final study. During the five-year follow-up period, the overall prevalence of NAFLD was $8.7 \%$. across the quintile 1,2 , 3,4 and 5 of UHR, the prevalence of NAFLD among lean patients was increased from $2.4 \%, 5 \%, 7.9 \%$, $10.3 \%$ to $17.8 \%$. After adjustment for age, markers of liver and kidney function, gender and metabolic indicators, multivariate cox proportional hazard regression analysis demonstrated that the hazard ratio(HR) was 1.99(1.43-2.73) in highest UHR (quintile 5) compared with lowest UHR(quintile 1). The area under the curve(AUC) of UHR (0.690) was higher than in UA (0.666) and HDL-C (0.636), which showed that the predictive ability of the UHR to new-onset NAFLD was better than serum uric acid and HDL-C. Even within the normal range of UA and HDL-c levels, UHR was independently associated with NAFLD, and HR (95\% confidence interva, $95 \% \mathrm{Cl})$ for NAFLD in Quintile 5 of UHR was 6.74(4.32-10.53). Compared with other significant predictors, the AUC value of UHR(0.67) was similar to that of low-density lipoprotein cholesterol(LDL-c)/HDL-c ratio(0.68), non-high-density lipoprotein cholesterol (NHDL-c)/HDL-c ratio(0.68) and alanine aminotransferase (ALT)/ aspartate aminotransferase (AST) ratio(0.7), and superior to that of LDL-c (0.63), remnant cholesterol (RC,0.59), albumin(ALB)/alkaline phosphatase(ALP) ratio(0.61). The sensitivity of UHR (70.5\%) was the highest among all indicators. In the subgroup of ALT, the AUC of UHR was 0.70 , which was the highest among all predictors in subjects with ALT $<40$. For subjects with elevated ALT levels (ALT > $40 \mathrm{U} / L)$, there was no statistical significance among RC $(P=0.441), A L B / A L P$ $(P=0.419)$ and ALT/AST $(P=0.159)$. In contrast, UHR's performance in predicting NAFLD was meaningful and reliable $(A \cup C=0.61, p<0.001)$.

Conclusions: UHR serve as an inexpensive and reliable predictor of NAFLD in lean Chinese people with normal blood lipid levels. It can be used to identify people at high risk of NAFLD.

\section{Background}

Millions of people worldwide suffer from liver disease, with advances in modern medicine, the prevalence of many liver diseases is declining. The number of new cases has also fallen sharply in many countries, including China. However, with the improvement of living standards, the incidence of nonalcoholic fatty liver disease (NAFLD) is increasing every year ${ }^{[1]}$. NAFLD is characterized by lipid deposits in liver cells, 
including hepatic steatosis, steatohepatitis, fibrosis and cirrhosis ${ }^{[2]}$. It affects nearly a third of adults worldwide and is particularly severe in developed countries ${ }^{[3]}$. NAFLD has become a new challenge for China. From 2003 to 2012, the prevalence of NAFLD increased from $17 \%$ to $22 \%$, comparable to the United States and Europe, according to statistics ${ }^{[1,4]}$. At present, liver biopsy is the gold standard for evaluating liver injury in NAFLD, but it is difficult to be widely carried out in clinical due to various limitations. Therefore, many studies hope to find simpler and valuable predictive indicators through different serum markers. Recent a study has proposed that serum uric acid (UA) may be a predictor of the severity of liver damage in NAFLD ${ }^{[5]}$. However, current information on the association of serum uric acid to high-density lipoprotein cholesterol(HDL-c) ratio (UHR) with new-onset NAFLD among the lean population with normal blood lipid levels is limited. Given this, our study was performed to demonstrate whether UHR could be used to independently assess risk of NAFLD in lean people with normal lipids and to analyze its predictive value.

\section{Methods}

\section{Study design and population}

This study was a secondary analysis of a longitudinal cohort study which enrolled 16,173 lean Chinese People in Wenzhou People's Hospital from January 2010 to December 2014 . The data were from the Dryad database and shared by Sun et al[ ${ }^{[6]}$. Exclusion criteria at baseline has been mentioned in previous studies. In addition, patients with dyslipidemia (total cholesterol (TC) $>5.2 \mathrm{mmol} / \mathrm{L}$, triglyceride (TG) $>1.7$ $\mathrm{mmol} / \mathrm{L}$, low-density lipoprotein cholesterol (LDL-C) $>3.12 \mathrm{mmol} / \mathrm{L}, \mathrm{HDL}-\mathrm{C}<1.03 \mathrm{mmol} / \mathrm{L}$ ) were excluded; A total of 9838 subjects were enrolled in this study. The research ethics had been authorized in the former study and this study did not need to apply for ethical approval again.

\section{Data collection}

The trained physician would deliver the standardized self-filling spreadsheet to collect the detailed information on basic clinical data冈such as height, weight, gender, age and body mass index (BMI) was calculated as weight divided by height. Blood pressure was measured and recorded by the professional investigators using an automated sphygmomanometer. All values of biochemical measurements were measured by an automated analyser (Abbott AxSYM) using standard methods. The laboratory parameters included TC, TG, LDL-C, HDL-C, UA, blood urea nitrogen (BUN), creatinine(Cr), alanine aminotransferase(ALT), aspartate aminotransferase (AST), albumin (ALB), fasting plasma glucose (FPG), total bilirubin (TB), globulin (GLB), total protein (TP).

\section{Diagnosis of NAFLD and follow-up}

During the 5-year follow-up period, subjects were evaluated annually by hepatic ultrasonic examinations. Diagnosis of NAFLD was based on criteria suggested by the Chinese Liver Disease Association in $2010^{[7]}$. The contents included diffuse hyperechogenicity of the liver compared to spleen and kidney, combined 
with any of the following: i) unclear display of intrahepatic structure; ii) enlarged liver with a round and blunt border; iii)The right liver lobe and diaphragm are unclear or incomplete; and iv) weakening of hepatic blood flow signal, but the distribution of bloodflow is normal. UA/HDL-c ratio was calculated as serum uric acid levels(mmol/L) divided by HDL-c levels ( $\mathrm{mmol} / \mathrm{L})$.

\section{Statistical analysis}

To assess the association between UHR and prevalence of NAFLD, all subjects were divided into five quintile groups as follows: Q1: $\leq 120.88$; Q2: 120.89-154.01; Q3: 154.02-189.91; Q4: 189.92-240.46; Q5: $\geq 240.47$. Baseline characteristics were described and compared. Continuous variables are summarized as mean \pm standard deviation(SD), and categorical variables are displayed as median (quartile). The significance of differences between groups was evaluated by a non-parametric test and a one-way analysis of variance(ANOVA) for continuous variable and $\chi 2$ test for categorical variables. $P$ values $<0.05$ (double-tailed) was considered statistically significant. Kaplan-meier analysis was used to calculate the cumulative hazard of NAFLD over time. The hazard ratios(HRs) and $95 \%$ confidence intervals $(\mathrm{Cl})$ based on the Cox proportional hazards regression model. Five models were used in this study, and model 1 was the original model without adjustment. Model 2 was adjusted only for age and gender. Model 3 adjusted for model 2 plus major markers of liver and kidney function (ALP, ALT, AST, ALB, TP, ALB, TB, BUN and Cr). Model 4 adjusted for model 3 plus metabolic indicators (FPG, TC, TG, LDL, BMI and SBP). UA and HDL were excluded from the model to avoid potential confusability effects since UA and HDL were included in UHR. Receiver operator characteristic (ROC) curve analyses were performed to evaluate the best model. A sensitivity analysis tests were performed to exclude the interaction effect between UA and HDL levels on UHR. In order to assess the abilities of UHR to detect NAFLD. UHR was compared to other significant predictors that have been proposed recently, such as LDL-C /HDL-c ratio, ALB/ALP ratio, ALT/AST ratio, non-HDL cholesterol(NHDL-c) to HDL-c ratio and remnant cholesterol (RC). The ability to diagnosis of NAFLD by ROC curve analyses. Finally, considering that the correlation between the above indicators and NAFLD may be affected by different conditions, some subgroup stratified analyses (gender and ALT) were conducted. All data were evaluated using SPSS 22.0 (SPSS Inc., Chicago, IL, USA).

\section{Results}

\section{Characteristics of study subjects}

Among the 16,173 Lean chinese participants enrolled in the study. Through inclusion and exclusion criteria, a total of 9,838 lean patients with normal blood lipid levels were included in the final study. The study subjects mean age was $42.5 \pm 14.7$ years old, the proportion of male and female patients was almost equal (51.4\% VS 48.6\%). During the five-year follow-up period, the overall prevalence rate of NAFLD was $8.7 \%$. the subjects were divided into five quintile groups according to UHR and the basic characteristics of five groups in table1. The subjects with high UHR levels had higher age, weight, height, DBP, SBP, BMI, LDL-C, ALT, AST, TP, TB, TC, TG, ALP, ALB, BUN, UA, Cr and FPG, while GLB, TC and HDL-C were lower. 


\section{Association between UHR and new-onset NAFLD.}

In Table1 and Fig.1, across the quintile 1, 2, 3, 4 and 5 of UHR, the prevalence of NAFLD among lean patients was increased from $2.4 \%, 5 \%, 7.9 \%, 10.3 \%$ to $17.8 \%$. The Kaplan-Meier analysis showed that increased UHR level was positively correlated with the cumulative incidence of NAFLD. This trend suggests that higher UHR levels are associated with higher prevalence of NAFLD. Table2 summarizes the analysis results of the multivariate cox proportional hazard regression. In unadjusted model1, we found a positive correlation between UHR and new-onset NAFLD. After adjustment for age and gender (model 2), and further for ALP, ALT, AST, ALB, TP, ALB, TB, BUN and $\mathrm{Cr}$ (model 3), The positive correlation did not change and less variation between quintile groups. Finally, after further adjusting for FPG, TC, TG, LDL, $\mathrm{BMI}$ and SBP(model 4), Compared with quintile 1, the HR(95\% Cl) for new-onset NAFLD in the increasing quintiles of UHR (Q2, Q3, Q4 and Q5) were 1.44(1.02-2.04), 1.48(1.06-2.06), 1.69(1.22-2.34) and 1.99(1.43-2.73), p<0.001,respectively. As shown in Fig.2, the results of ROC curve analyses showed that model 4 is the best model to detect NAFLD, the area under receiver operator characteristic curve(AUC) of model 4 was $0.723(p<0.001)$.

\section{Association between UA, HDL-c and UHR.}

A ROC curve analyses to evaluate the ability of serum uric acid, HDL cholesterol and the UA/HDL-c ratio to predict the risk of new-onset NAFLD. The AUC of UHR was $0.690(0.672-0.708)$, which is higher than 0.666 (0.647-0.684) in UA and $0.636(0.617-0.654)$ in HDL-c(Fig.3). It showed that the predictive ability of the UHR to new-onset NAFLD was better than UA and HDL-c. At the same time, participants with hyperuricemia ( $U A>420 \mu \mathrm{mol} / \mathrm{L}$ in male and $>360 \mu \mathrm{mol} / \mathrm{L}$ in female) and low high-density lipoprotein cholesterolemia (HDL-cholesterol $<1.03 \mathrm{mmol} / \mathrm{L}$ ) were further excluded from the initial enrollment and sensitivity analysis tests were performed. Among 5,357 participants with normal serum uric acid and high-density lipoprotein cholesterol levels, 518 lean patients had new-onset NAFLD. As shown in Table 3, even within the normal range of UA and HDL-c levels, UHR was independently associated with NAFLD, and HR $(95 \% \mathrm{Cl})$ for NAFLD in Quintile 5 of UHR was 6.74(4.32-10.53).

\section{UHR and other significant predictors}

The difference in the ability of UHR and other significant predictors in detecting new-onset NAFLD in lean Chinese adults was tested by ROC curve analyses. In table 4, the AUC value of UHR and other seven predictors indices were greater than 0.5 , showing that all indicators have certain predictive ability. NAFLD. Furthermore, the AUC value of UHR(0.67) was similar to that of LDL-c/HDL-c ratio(0.68), NHDL-c/HDL-c ratio(0.68) and Alt/AST ratio(0.7), and superior to that of LDL (0.63), RC (0.59) and ALB/ALP ratio(0.61). The sensitivity of UHR (70.5\%) was the highest among all indicators, with a specificity of $57.3 \%$ and cutoff point of 179.9. In the subgroup of gender (Fig.4), the core outcome of the association between the significant predictors and NAFLD risk did not change. Compared with males, the AUC of the abovementioned indicators were greater in females (AUC of the UHR was 0.65 in males and 0.69 in females). In another subgroup of ALT (Fig.5), the AUC of UHR was 0.70, which was the highest among all predictors in subjects with $A L T<40$. For subjects with elevated ALT levels (ALT $>40 \mathrm{U} / \mathrm{L})$, there was no statistical 
significance among $R C(P=0.441)$, ALB/ALP ratio $(P=0.419)$ and ALT/AST ratio( $P=0.159)$. In contrast, UHR's performance in predicting NAFLD was meaningful and reliable (AUC $=0.61, p<0.001)$. Therefore, UHR is a Valuable predictor to detect new-onset NAFLD.

\section{Discussion}

In this 5-year longitudinal cohort study, we found that UHR has significant positive correlation with NAFLD in lean Chinese people with normal blood lipid levels. According to quintile 1 to 5 of UHR, the result of Kaplan-Meier analysis and multivariate cox proportional hazard regression analysis were shown that the Chinese population with elevated UHR had higher risk and cumulative incidence of NAFLD among lean individuals with normal lipid levels. Meanwhile, the relationship between UHR and NAFLD was independent of other variates. The rising global incidence of NAFLD has been associated with obesity and metabolic syndrome in the past ${ }^{[8]}$. But recently, NAFLD has become more common in nonobese people, especially in China ${ }^{[9]}$.Sun et al. ${ }^{[6]}$ found that the association of LDH-c within the normal range and NAFLD in the non-obese Chinese population for the first time. To date, the risk factors for NAFLD in lean adults have not been elucidated. Kosekli et al. proposed that UHR might serve as a novel and reliable marker for NAFLD. This was consistent with the main results of this study, but we focus on the association between non-obese patients with normal lipid levels and the prevalence of NAFLD ${ }^{[3]}$. Recently, a new terminology, metabolic dysfunction-associated fatty liver disease (MAFLD), has been widely discussed. Although its utility requires further confirmation, it has been proved that metabolic disorders played a significant role in the occurrence of NAFLD ${ }^{[10]}$. Serum uric acid is a product of purine metabolism in body, which is closely related to metabolic diseases such as gout, cardiovascular disease, hypertriglyceridemia and diabetes mellitus ${ }^{[11-14]}$. The relationship between NAFLD and serum uric acid level was first mentioned in $2002^{[15]}$. Since then, some studies have confirmed that UA was an independent risk factor for NAFLD ${ }^{[16,17]}$ and level of serum uric acid was positively correlated with histological damage ${ }^{[18]}$. Each $1 \mathrm{mg} / \mathrm{dL}$ UA level increment, twenty-one percent increase in the risk of NAFLD ${ }^{[19]}$. Hyperuricemia promotes metabolic disorder and prevalence of NAFLD through various mechanisms including insulin resistance (IR), oxidative stress, and fructose metabolism disorder ${ }^{[20,21]}$. There was evidence that HDL-c was involved in metabolic syndrome and NAFLD patients had low HDL cholesterolemia usually ${ }^{[22]}$. In this study, through ROC curve analysis, we found that the predictive ability of the ratio of UA to HDL-c was higher than that of UA and HDL alone. Moreover, UHR was independently associated with NAFLD even within the normal range of UA and HDL-C levels, which greatly increases the clinical application value. Our results are consistent with Zhang et al ${ }^{[23]}$. In addition to sensitivity analysis, we also compared UHR with recently proposed indicators for predicting new-onset NAFLD among lean adults ${ }^{[24-28]}$. The results showed that UHR was excellent in predictive value and sensitivity. Subgroup analysis was performed to determine whether there were differences between genders and levels of liver function. Finally, we found that gender had little effect on each predictor, but abnormal liver function had different effect on each indicator. In contrast, the predictive value of UHR was stable and applicable in patients with abnormal liver function. 
This is a large sample retrospective cohort study. As we know, this is the first study to demonstrate the association between UHR and new-onset NAFLD in Lean Chinese People with normal blood lipid levels. This study has the following limitations: Frist, this study was designed retrospectively, which could let to results of the study less prospective predictive value. Secondly, the object of this study is the Chinese population, which differ from other ethnic groups in some indicators and definitions (such as BMI).

Finally, the recently proposed new concept of MAFLD, whose diagnostic criteria were not used in this study ${ }^{[29]}$. Therefore, the correlation cannot be further confirmed.

\section{Conclusion}

UHR is independently associated with NAFLD in lean Chinese people with normal blood lipid levels or normal serum uric acid levels. UHR is an inexpensive and reliable predictor to detect new-onset NAFLD.

\section{Abbreviations}




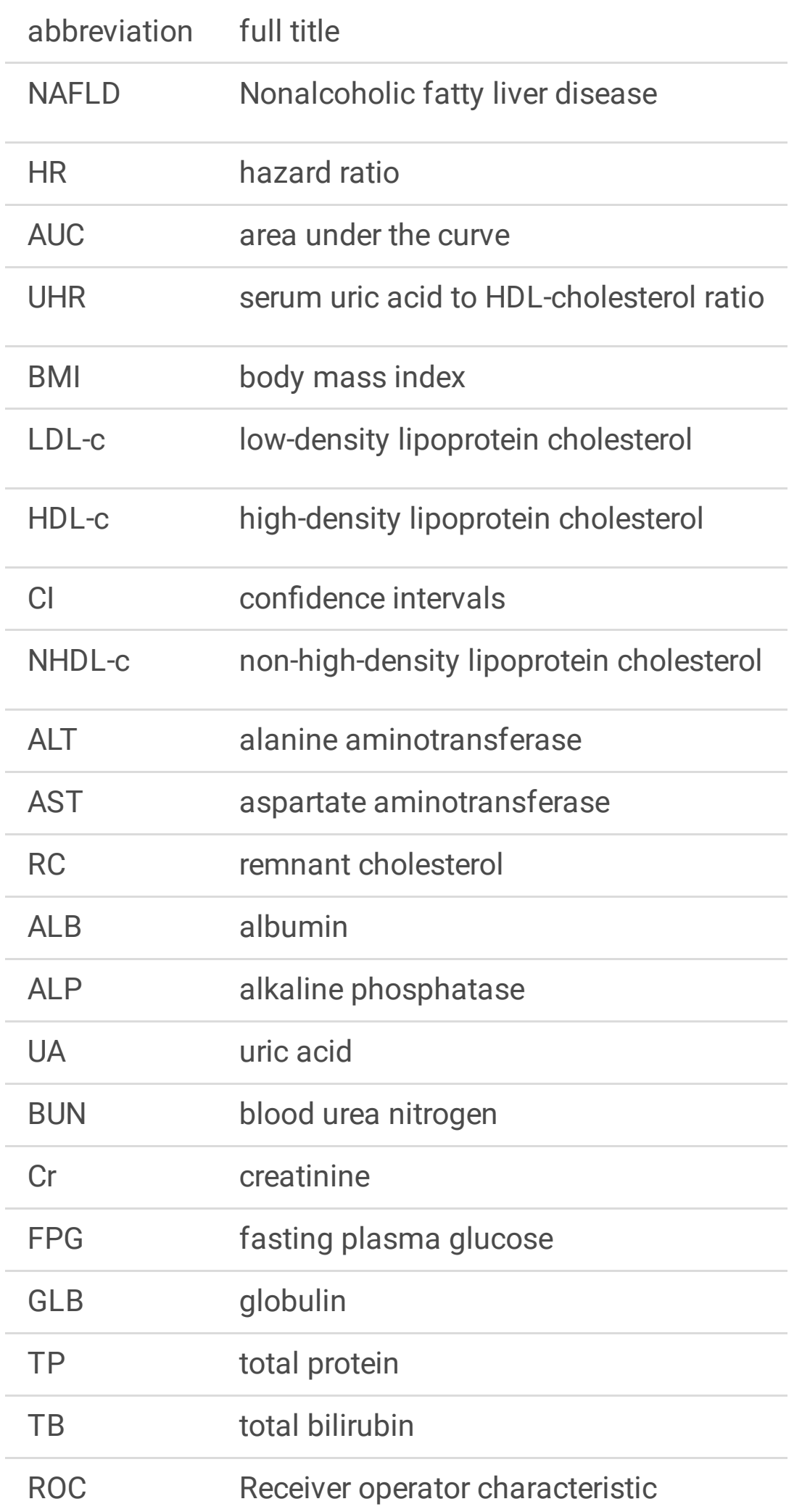

\section{Declarations}

Acknowledgements

Thanks to Dr Sun's team for sharing study data. 


\section{Authors'contributions}

Conceptualization and research design: Xiaoping Wu and Wentao Zhu. Supervision: Xiaoping Wu, Song Yuan and Ying Zhu; Project administration: Pei Shi and An Liang; Writing-Original draft preparation:

Wentao Zhu; Writing- Reviewing and Editing: Xiaoping Wu, Wentao Zhu, An Liang , Jiwei Fu, Zhilong Wen and Ting Zheng. The author(s) read and approved the final manuscript.

\section{Funding}

Not applicable.

\section{Availability of data and materials}

Datasets that support the conclusions of this article were available in the [DRYAD] repository. [https://datadryad.org].

\section{Ethics approval and consent to participate}

Since this study is a post-hoc analysis of previous study, and Wenzhou people's Hospital approved the research plan. this study did not need to apply for ethical approval again.

\section{Consent for publication}

Not applicable.

\section{Competing interests}

The authors declare that they have no conflicts of interest.

\section{References}

1. Xiao J, Wang F, Wong NK, He J, Zhang R, Sun R, Xu Y, Liu Y, Li W, Koike K, He W, You H, Miao Y, Liu X, Meng M, Gao B, Wang H, Li C. Global liver disease burdens and research trends: Analysis from a Chinese perspective. J Hepatol. 2019 Jul;71(1):212-221. doi: 10.1016/j.jhep.2019.03.004. Epub 2019 Mar 12. PMID: 30871980.

2. ]Hijona E, Hijona L, Arenas JI, Bujanda L. Inflammatory mediators of hepatic steatosis. Mediators Inflamm. 2010;2010:837419. doi: 10.1155/2010/837419. Epub 2010 Mar 16. PMID: 20300479; PMCID: PMC2840375.

3. Kosekli MA, Kurtkulagii O, Kahveci G, Duman TT, Tel BMA, Bilgin S, Demirkol ME, Aktas G. The association between serum uric acid to high density lipoprotein-cholesterol ratio and non-alcoholic fatty liver disease: the abund study. Rev Assoc Med Bras (1992). 2021 Apr;67(4):549-554. doi: 10.1590/1806-9282.20201005. PMID: 34495059. 
4. Wang FS, Fan JG, Zhang Z, Gao B, Wang HY. The global burden of liver disease: the major impact of China. Hepatology. 2014 Dec;60(6):2099-108. doi: 10.1002/hep.27406. Epub 2014 Oct 29. PMID: $25164003 ;$ PMCID: PMC4867229.

5. Lombardi R, Pisano G, Fargion S. Role of Serum Uric Acid and Ferritin in the Development and Progression of NAFLD. Int J Mol Sci. 2016 Apr 12;17(4):548. doi: 10.3390/ijms17040548. PMID: $27077854 ;$ PMCID: PMC4849004.

6. Sun DQ, Wu SJ, Liu WY, Wang LR, Chen YR, Zhang DC, Braddock M, Shi KQ, Song D, Zheng MH. Association of low-density lipoprotein cholesterol within the normal range and NAFLD in the nonobese Chinese population: a cross-sectional and longitudinal study. BMJ Open. 2016 Dec 7;6(12):e013781. doi: 10.1136/bmjopen-2016-013781. PMID: 27927668; PMCID: PMC5168665.

7. Zeng MD, Fan JG, Lu LG, Li YM, Chen CW, Wang BY, Mao YM; Chinese National Consensus Workshop on Nonalcoholic Fatty Liver Disease. Guidelines for the diagnosis and treatment of nonalcoholic fatty liver diseases. J Dig Dis. 2008 May;9(2):108-12. doi: 10.1111/j.1751-2980.2008.00331.x. PMID: 18419645.

8. Khashab MA, Liangpunsakul S, Chalasani N. Nonalcoholic fatty liver disease as a component of the metabolic syndrome. Curr Gastroenterol Rep. 2008 Feb;10(1):73-80. doi: 10.1007/s11894-008-00120. PMID: 18417046.

9. Liu CJ. Prevalence and risk factors for non-alcoholic fatty liver disease in Asian people who are not obese. J Gastroenterol Hepatol. 2012 Oct;27(10):1555-60. doi: 10.1111/j.1440-1746.2012.07222.x. PMID: 22741595.

10. Huang Q, Zou X, Wen X, Zhou X, Ji L. NAFLD or MAFLD: Which Has Closer Association With AllCause and Cause-Specific Mortality?-Results From NHANES III. Front Med (Lausanne). $2021 \mathrm{Jul}$ 1;8:693507. doi: 10.3389/fmed.2021.693507. PMID: 34277667; PMCID: PMC8280321.

11. Zhang ML, Gao YX, Wang X, Chang H, Huang GW. Serum uric acid and appropriate cutoff value for prediction of metabolic syndrome among Chinese adults. J Clin Biochem Nutr. 2013 Jan;52(1):38-42. doi: 10.3164/jcbn.12-65. Epub 2012 Oct 24. PMID: $23341696 ;$ PMCID: PMC3541417.

12. Choi HK, Ford ES. Prevalence of the metabolic syndrome in individuals with hyperuricemia. Am J Med. 2007 May;120(5):442-7. doi: 10.1016/j.amjmed.2006.06.040. PMID: 17466656.

13. Kodama S, Saito K, Yachi Y, Asumi M, Sugawara A, Totsuka K, Saito A, Sone H. Association between serum uric acid and development of type 2 diabetes. Diabetes Care. 2009 Sep;32(9):1737-42. doi: 10.2337/dc09-0288. Epub 2009 Jun 23. PMID: 19549729; PMCID: PMC2732137.

14. Nejatinamini S, Ataie-Jafari A, Qorbani M, Nikoohemat S, Kelishadi R, Asayesh H, Hosseini S. Association between serum uric acid level and metabolic syndrome components. J Diabetes Metab Disord. 2015 Sep 14;14:70. doi: 10.1186/s40200-015-0200-z. PMID: 26380228; PMCID: PMC4570526.

15. Lonardo A, Loria P, Leonardi F, Borsatti A, Neri P, Pulvirenti M, Verrone AM, Bagni A, Bertolotti M, Ganazzi D, Carulli N; POLI.ST.E.N.A. Study Group. Policentrica Steatosi Epatica Non Alcolica. Fasting insulin and uric acid levels but not indices of iron metabolism are independent predictors of non- 
alcoholic fatty liver disease. A case-control study. Dig Liver Dis. 2002 Mar;34(3):204-11. doi: 10.1016/s1590-8658(02)80194-3. PMID: 11990393.

16. Fernández Rodríguez CM, Aller R, Gutiérrez García ML, Ampuero J, Gómez-Camarero J, MartínMateos RMa , Burgos-Santamaría D, Rosales JM, Aspichueta P, Buque X, Latorre M, Andrade RJ, Hernández-Guerra M, Romero-Gómez M. Higher levels of serum uric acid influences hepatic damage in patients with non-alcoholic fatty liver disease (NAFLD). Rev Esp Enferm Dig. 2019 Apr;111(4):264269. doi: 10.17235/reed.2019.5965/2018. PMID: 30810330.

17. Li Y, Xu C, Yu C, Xu L, Miao M. Association of serum uric acid level with non-alcoholic fatty liver disease: a cross-sectional study. J Hepatol. 2009 May;50(5):1029-34. doi: 10.1016/j.jhep.2008.11.021. Epub 2009 Jan 9. PMID: 19299029.

18. Petta $S$, Cammà C, Cabibi D, Di Marco V, Craxì A. Hyperuricemia is associated with histological liver damage in patients with non-alcoholic fatty liver disease. Aliment Pharmacol Ther. 2011 Oct;34(7):757-66. doi: 10.1111/j.1365-2036.2011.04788.x. Epub 2011 Jul 25. PMID: 21790685.

19. Yuan H, Yu C, Li X, Sun L, Zhu X, Zhao C, Zhang Z, Yang Z. Serum Uric Acid Levels and Risk of Metabolic Syndrome: A Dose-Response Meta-Analysis of Prospective Studies. J Clin Endocrinol Metab. 2015 Nov;100(11):4198-207. doi: 10.1210/jc.2015-2527. Epub 2015 Aug 26. PMID: 26308292.

20. Zheng X, Gong L, Luo R, Chen H, Peng B, Ren W, Wang Y. Serum uric acid and non-alcoholic fatty liver disease in non-obesity Chinese adults. Lipids Health Dis. 2017 Oct 16;16(1):202. doi:

10.1186/s12944-017-0531-5. PMID: 29037239; PMCID: PMC5644248.

21. Abreu E, Fonseca MJ, Santos AC. Associação entre a hiperuricemia e a resistência à insulina [Association between hyperuricemia and insulin resistance]. Acta Med Port. 2011 Dec;24 Suppl 2:565-74. Portuguese. Epub 2011 Dec 31. PMID: 22849948.

22. Sumner AE, Zhou J, Doumatey A, Imoisili OE, Amoah A, Acheampong J, Oli J, Johnson T, Adebamowo C, Rotimi CN. Low HDL-Cholesterol with Normal Triglyceride Levels is the Most Common Lipid Pattern in West Africans and African Americans with Metabolic Syndrome: Implications for Cardiovascular Disease Prevention. CVD Prev Control. 2010 Sep 1;5(3):75-80. doi: 10.1016/j.cvdpc.2010.07.003. PMID: 21113431; PMCID: PMC2989612.

23. Zhang YN, Wang QQ, Chen YS, Shen C, Xu CF. Association between Serum Uric Acid to HDLCholesterol Ratio and Nonalcoholic Fatty Liver Disease in Lean Chinese Adults. Int J Endocrinol. 2020 Mar 23;2020:5953461. doi: 10.1155/2020/5953461. PMID: 32273892; PMCID: PMC7125489.

24. Zou Y, Lan J, Zhong Y, Yang S, Zhang H, Xie G. Association of remnant cholesterol with nonalcoholic fatty liver disease: a general population-based study. Lipids Health Dis. 2021 Oct 17;20(1):139. doi: 10.1186/s12944-021-01573-y. PMID: 34657611; PMCID: PMC8520640.

25. Zou Y, Zhong L, Hu C, Sheng G. Association between the alanine aminotransferase/aspartate aminotransferase ratio and new-onset non-alcoholic fatty liver disease in a nonobese Chinese population: a population-based longitudinal study. Lipids Health Dis. 2020 Nov 25;19(1):245. doi: 10.1186/s12944-020-01419-z. PMID: 33239040; PMCID: PMC7690093. 
26. Sheng G, Peng N, Hu C, Zhong L, Zhong M, Zou Y. The albumin-to-alkaline phosphatase ratio as an independent predictor of future non-alcoholic fatty liver disease in a 5-year longitudinal cohort study of a non-obese Chinese population. Lipids Health Dis. 2021 May 16;20(1):50. doi: 10.1186/s12944021-01479-9. PMID: 33993872; PMCID: PMC8126124.

27. Zou Y, Zhong L, Hu C, Zhong M, Peng N, Sheng G. LDL/HDL cholesterol ratio is associated with newonset NAFLD in Chinese non-obese people with normal lipids: a 5-year longitudinal cohort study. Lipids Health Dis. 2021 Mar 25;20(1):28. doi: 10.1186/s12944-021-01457-1. PMID: 33766067; PMCID: PMC7993485.

28. Yang S, Zhong J, Ye M, Miao L, Lu G, Xu C, Xue Z, Zhou X. Association between the non-HDLcholesterol to HDL-cholesterol ratio and non-alcoholic fatty liver disease in Chinese children and adolescents: a large single-center cross-sectional study. Lipids Health Dis. 2020 Nov 22;19(1):242. doi: 10.1186/s12944-020-01421-5. PMID: 33222696; PMCID: PMC7681973.

29. Lin S, Huang J, Wang M, Kumar R, Liu Y, Liu S, Wu Y, Wang X, Zhu Y. Comparison of MAFLD and NAFLD diagnostic criteria in real world. Liver Int. 2020 Sep;40(9):2082-2089. doi: 10.1111/liv.14548. Epub 2020 Jul 26. PMID: 32478487.

\section{Figures}




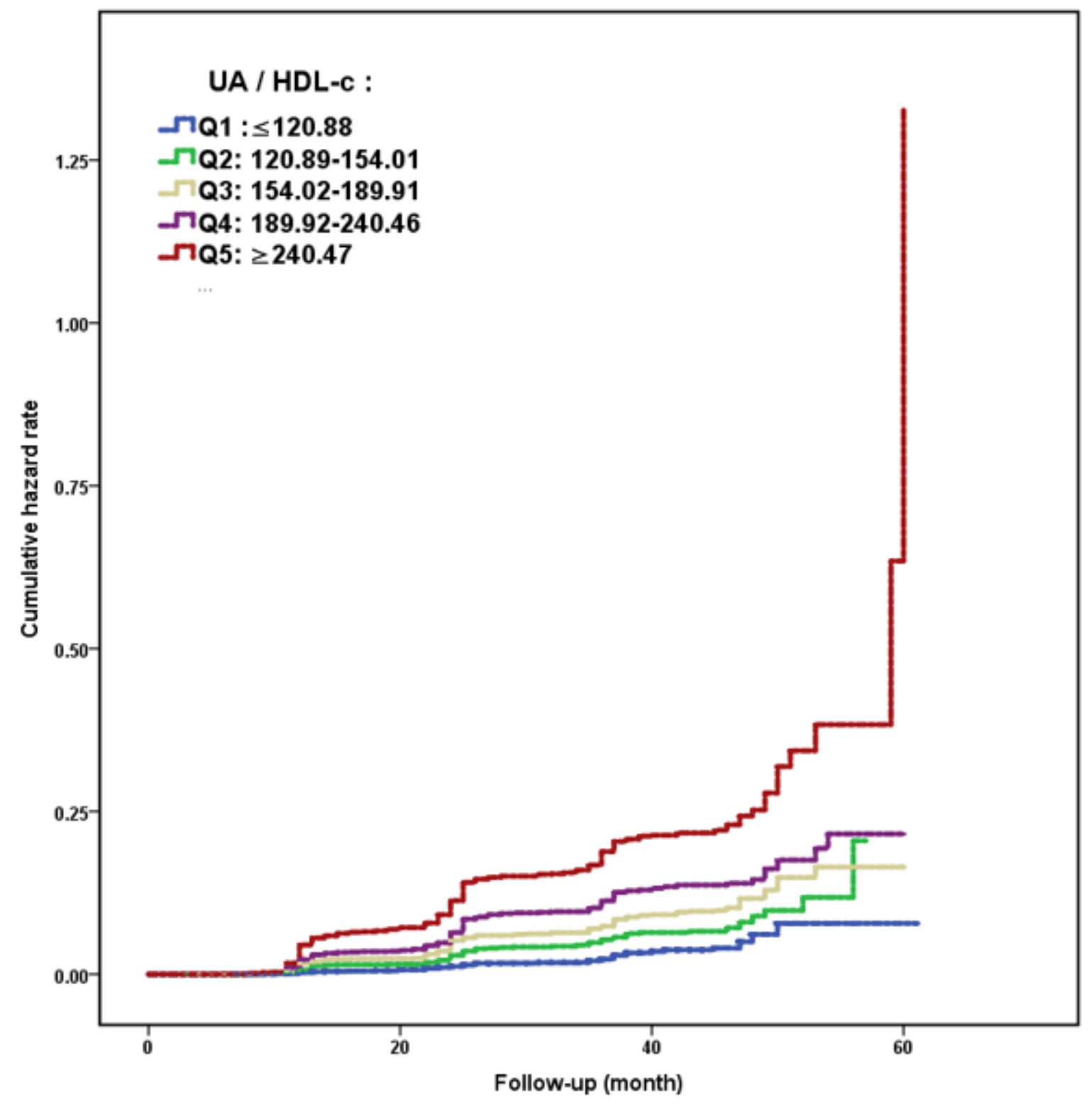

Figure 1

The Kaplan-Meier curves reflecting increased UA/HDL-c ratio level was positively correlated with the cumulative incidence of NAFLD in lean individuals with normal blood lipid levels. $P$ trend $<0.001$. HDL-c, highdensity lipoprotein cholesterol; UA, uric acid; 


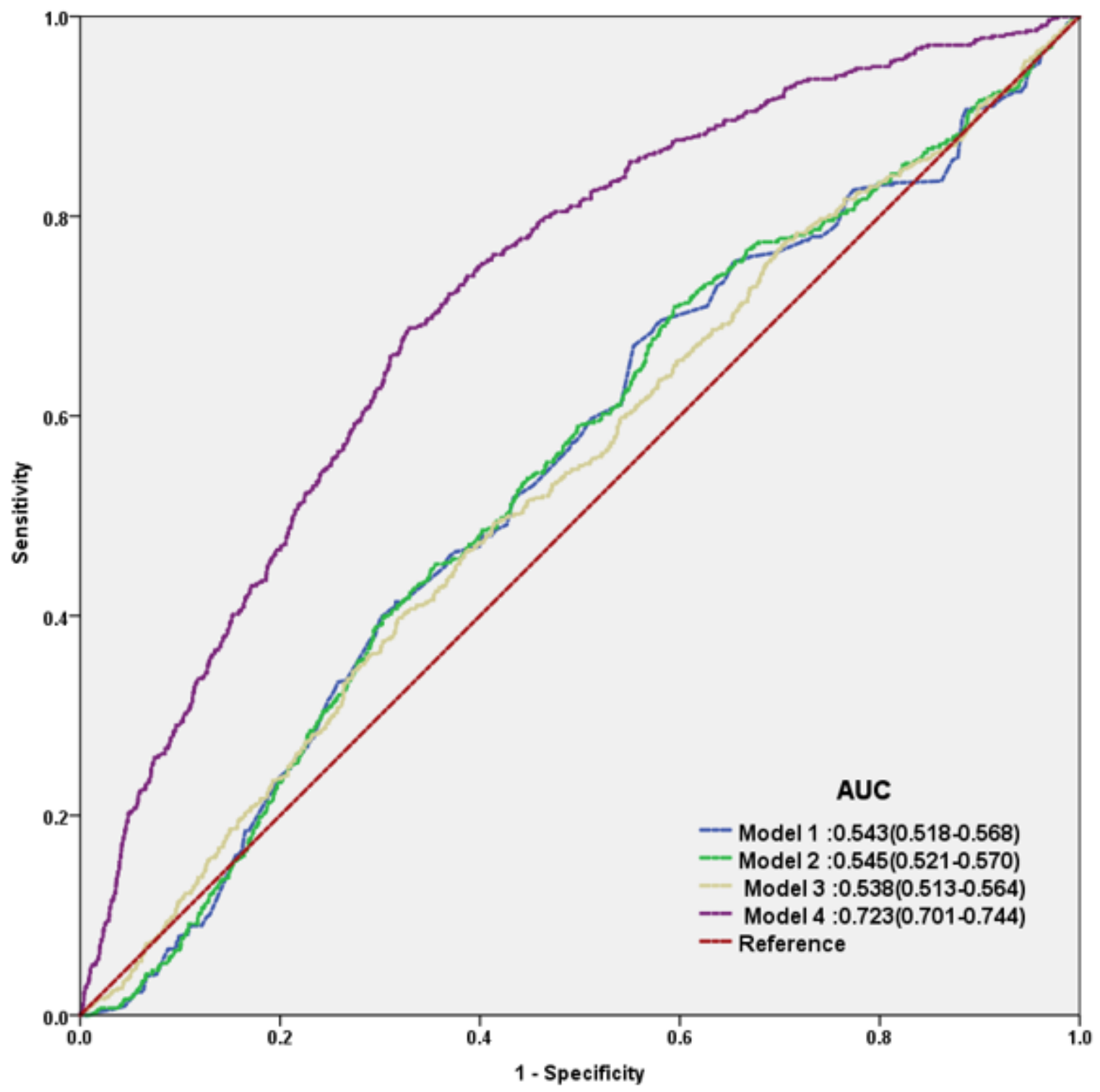

Figure 2

ROC curves of the five model, model 1 is unadjusted; Model 2 is adjusted for age, gender; Model 3 is further adjusted for markers of liver and kidney function; model 4 is further adjusted for metabolic markers. AUC, area under the curve; 


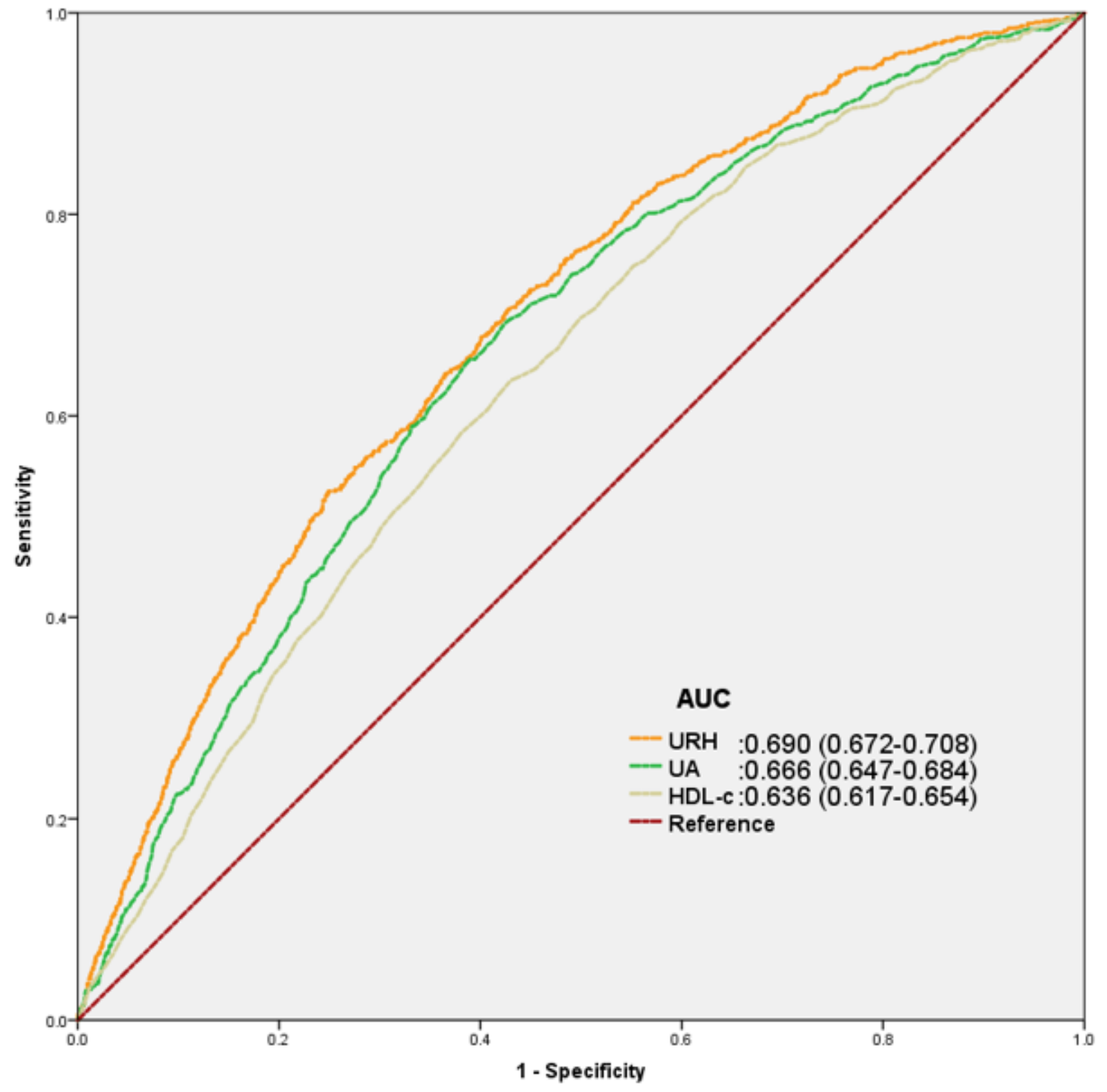

Figure 3

ROC curves of the UA/HDL-c ratio, UA and HDL-c. UA, uric acid; HDL-c, highdensity lipoprotein cholesterol; 

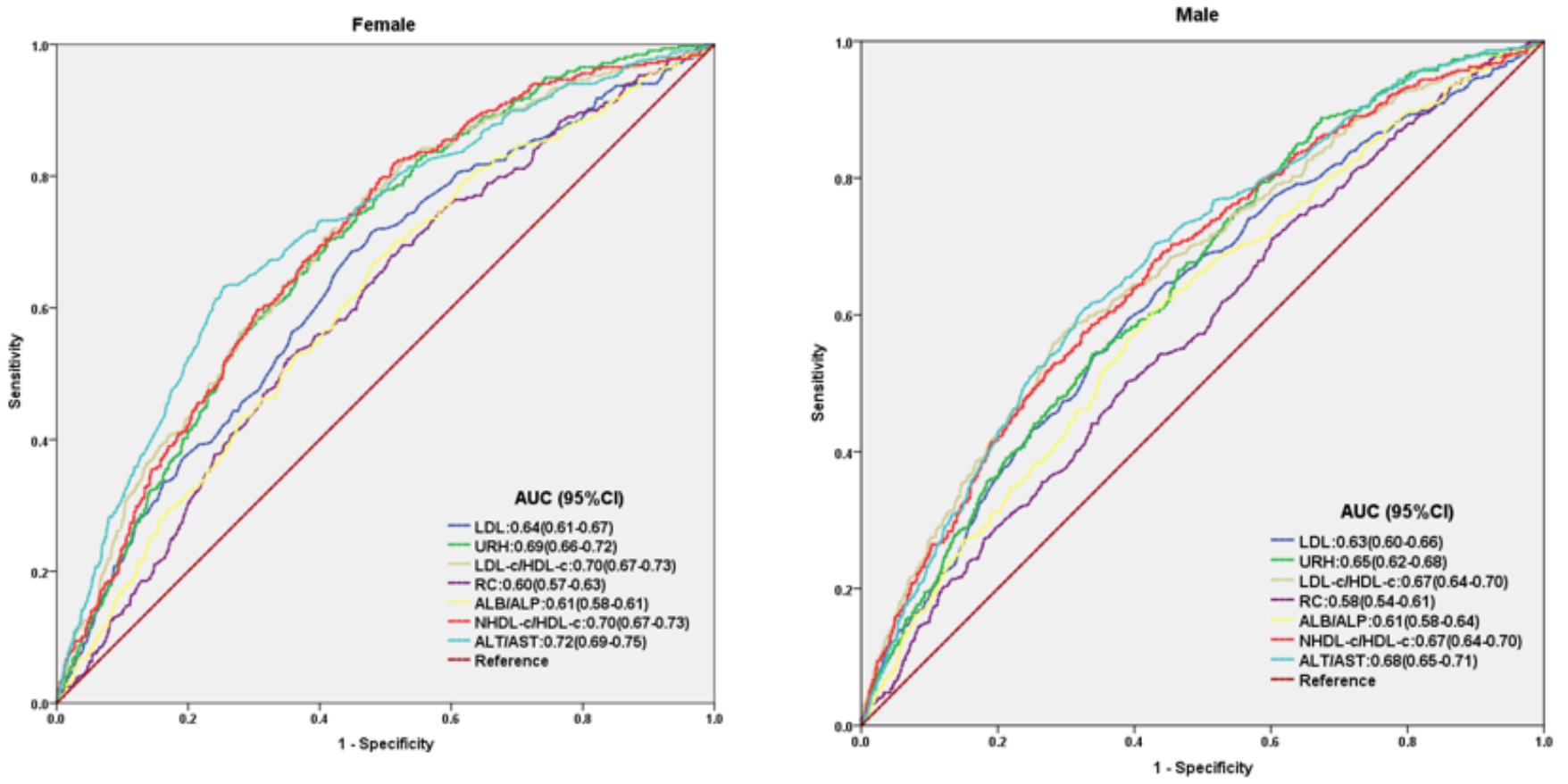

\section{Figure 4}

Comparison of the predictive value of NAFLD-related parameters for diagnosis of NAFLD among males and females.
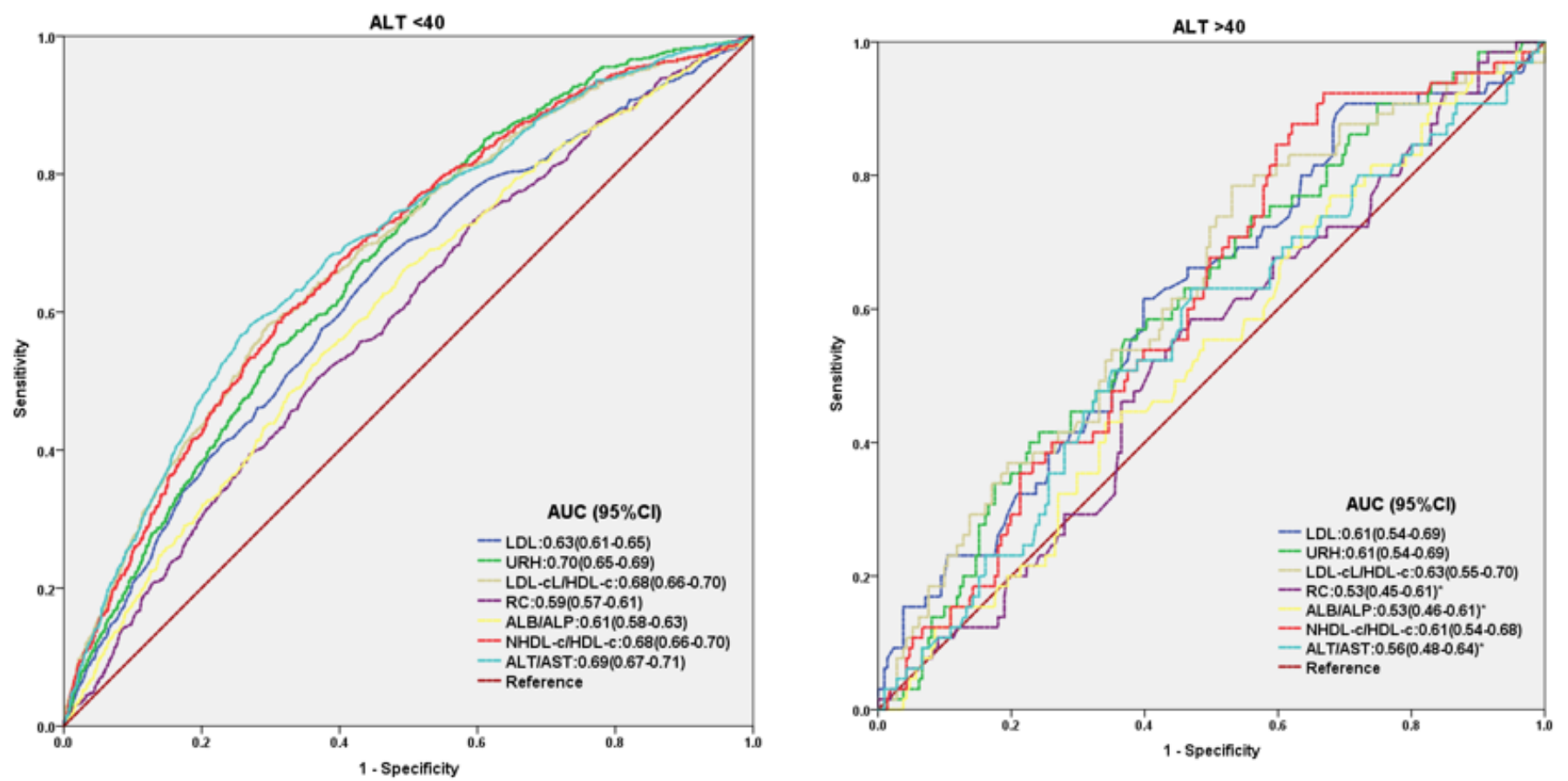

Figure 5 
ROC curves of NAFLD-related parameters in subjects among ALT $<40$ and ALT $>40$.

\section{Supplementary Files}

This is a list of supplementary files associated with this preprint. Click to download.

- table1.xlsx

- table2.xlsx

- table3.xlsx

- table4.xIsx 